\title{
CXCR7 receptors facilitate the progression of colon carcinoma within lung not within liver
}

\author{
E Guillemot $^{1,2}$, B Karimdjee-Soilihi ${ }^{1,2,3}$, E Pradelli ${ }^{1,2}$, M Benchetrit $^{1,4}$, E Goguet-Surmenian ${ }^{1,2}$, \\ M-A Millet ${ }^{1,2}$, F Larbret ${ }^{1,5}$, J-F Michiels ${ }^{1,4}$, D Birnbaum ${ }^{6}$, P Alemanno ${ }^{3}$, H Schmid-Antomarchi ${ }^{1,2,7}$ and \\ A Schmid-Alliana*,1,2,7
}

'Université de Nice Sophia-Antipolis, UFR Sciences, Nice 06108, France; ${ }^{2}$ FRE-CNRS 3472, Nice 06202, France; ${ }^{3}$ Polyclinique St Jean, Cagnes/mer 06800, France; ${ }^{4}$ Laboratoire Central d'Anatomie Pathologique, CHU Pasteur, Nice 06002, France; ${ }^{5}$ EA 6302, CHU Archet I, Nice 06202 , France; 6INSERM UMR599, Institut Paoli Calmettes, Marseille 13009, France

BACKGROUND: Liver and lung metastases are the predominant cause of colorectal cancer (CRC)-related mortality. Chemokinereceptor pairs have a critical role in determining the metastatic progression of tumours. Our hypothesis was that disruption of CXCR7/CXCR7 ligands axis could lead to a decrease in CRC metastases.

METHODS: Primary tumours and metastatic tissues from patients with CRC were tested for the expression of CXCR7 and its ligands. Relevance of CXCR7/CXCR7 ligands for CRC metastasis was then investigated in mice using small pharmacological CXCR7 antagonists and CRC cell lines of human and murine origins, which - injected into mice - enable the development of lung and liver metastases.

RESULTS: Following injection of CRC cells, mice treated daily with CXCR7 antagonists exhibited a significant reduction in lung metastases. However, CXCR7 antagonists failed to reduce the extent of liver metastasis. Moreover, there were subtle differences in the expression of CXCR7 and its ligands between lung and liver metastases.

CONCLUSION: Our study suggests that the activation of CXCR7 on tumour blood vessels by its ligands may facilitate the progression of CRC within lung but not within liver. Moreover, we provide evidence that targeting the CXCR7 axis may be beneficial to limit metastasis from colon cancer within the lungs.

British Journal of Cancer (2012) I 07, 1944-1949. doi:10.1038/bjc.2012.503 www.bjcancer.com

Published online 20 November 2012

(c) 2012 Cancer Research UK

Keywords: chemokines/receptors; CXCR7 antagonists; colon cancer; metastasis; animal models

Colorectal cancer (CRC) is one of the most common malignancies, and in spite of the conventional treatments, most of the CRC patients die from distant metastases, predominantly in the liver and lungs (Weitz et al, 2005). Thus, the control of metastasis represents the greatest clinical challenge in the treatment of colon cancer.

Although vascular drainage patterns may contribute to a mechanical process for distant metastasis, there is growing evidence pointing to a chemokine involvement in this process. To date, several pairs of chemokine/receptor have been identified to have key roles in cancer progression and organ-selective metastasis (Zlotnik et al, 2011; Mukaida and Baba, 2012). The best-studied and well-established example is that of the CXCL12 chemokine and its receptor CXCR4. This pair was shown to be involved in almost all malignancies studied, and in most cases, the expression of CXCR4 correlates with the degree of malignancy and metastasis formation (Sun et al, 2010). The CXCL12/CXCR4 axis is implicated in several aspects of tumour progression including migration, proliferation and/or survival of tumour and endothelial cells (Kryczek et al, 2007; Keeley et al, 2011).

\footnotetext{
*Correspondence: Dr A Schmid-Alliana;

E-mail: schmid@unice.fr

${ }^{7}$ These authors contributed equally to this work.

Received 6 July 2012; revised 10 October 2012; accepted 17 October 2012; published online 20 November 2012
}

In CRC patients, CXCR4 expression by colorectal tumour cells is significantly associated with lymphatic and liver dissemination, with an increased risk for tumour recurrence, as well as a poor prognosis (Verbeke et al, 2011). Moreover, it has been shown, in a mouse model of metastatic colon cancer, that blocking of the CXCR4 function on carcinoma cells induced a markedly decreased number of liver and lung metastases. According to the authors, this antitumor effect resulted from the impairment of proliferation rather than from an inhibition of invasion (Zeelenberg et al, 2003).

Besides CXCR4, CXCL12 was shown to recognise with high affinity orphan receptor CXCR7/RDC1 (Balabanian et al, 2005). Schall's group was the first to demonstrate an implication of CXCR7 in physiological and pathological processes, including cell growth/survival and adhesion, as well as promotion of tumour growth (Burns et al, 2006). More recently, numerous studies confirmed the critical role of CXCR7 in promoting the growth of several cancers in vivo (Miao et al, 2007; Wang et al, 2008; Bennani-Baiti et al, 2010; Zheng et al, 2010; Monnier et al, 2012).

Our study aimed precisely at getting new insights into the role played by CXCR7/ligand interactions in the metastatic development of CRC within two major target organs, lungs and liver. Using mouse models of metastatic colon cancer and small pharmacological CXCR7 antagonists, we tested the therapeutic potential of CXCR7-blocking strategy on both types of experimental metastases. Furthermore, in order to establish a link 
between animal models and human disease, primary malignant and metastatic tissues from human patients were tested for the expression of CXCR7 and its ligands. Together, our results define CXCR7 axis as a key mediator for lung metastasis of colon cancer and identify CXCR7-blocking approaches as promising strategies against lung metastases.

\section{MATERIAL AND METHODS}

\section{Animal studies and tumour cell lines}

Female BALB/c and SCID mice, 6-8-weeks-old, were purchased from Harlan (Gannat, France). Murine C26 colon carcinoma tumour cells derived from BALB/c mice (Corbett et al, 1975) were provided by Dr Mario Colombo (Istituto Nazionale per lo Studio e la Cura dei Tumori, Milan, Italy), and human HT29 colorectal adenocarcinoma cells were obtained from LGC Promochem (Molsheim, France). Colorectal cancer cell lines were maintained in McCoy's medium (Gibco-BRL Life Technologies, Cergy-Pontoise, France) supplemented with $10 \%$ heat-inactivated FCS (Gibco-BRL).

\section{Ethics statement}

All of the procedures involving animals and their care were conducted in accordance with institutional guidelines (Permit number A06-088-14) and after approval by the local ethics committee: Comité Institutionnel d'Éthique Pour l'Animal de Laboratoire (Permit number NCA 2008-02).

Human tumour tissues used in real-time PCR (RT-PCR) experiments were obtained from the Institut Paoli Calmettes (IPC, Marseille, France) and were collected from patients with invasive colon adenocarcinoma who underwent surgical biopsy or initial surgery at the IPC between 1987 and 2007. Each patient gave written informed consent and the study was approved by the IPC 'Comité d'Orientation Stratégique'.

Human tumour tissues used for immunohistochemistry were obtained from the 'Laboratoire Central d'Anatomie Pathologique' of Pasteur Hospital (Nice, France). All tumour samples were collected at the 'Centre Hospitalier Universitaire' (CHU) of Nice (France) between 2004 and 2009, after obtaining written informed consent according to study approved by the ethics committee of $\mathrm{CHU}$ of Nice.

\section{TaqMan RT-PCR experiments}

Total RNA, from human and mouse tissues, as well as cell lines, was extracted using RNeasy kit (Qiagen, Courtaboeuf, France) and transcribed into cDNA using the Superscript III enzyme (Invitrogen, Cergy-Pontoise, France). Real-time PCR was performed in an ABI PRISM 7900, as explained in detail in Supplementary Appendix.

\section{Mouse models of pulmonary and liver metastasis}

For the induction of metastases, C26 and HT29 cells were delivered, as previously described (Cambien et al, 2009), by intravenous injection, respectively, into BALB/c or SCID mice. The treatments were delivered to mice by intraperitoneal injections of $100 \mathrm{mg} \mathrm{kg}^{-1}$ of the CCX754/CCX771 compounds (Burns et al, 2006 ), or vehicle, once a day on days +5 to +12 (syngeneic C26 models) or on days +7 to +28 and then, on days +36 to +57 (immunodeficient HT29 models). Fifteen days after the injection of the C26 cells or 9 weeks after the injection of HT29 cells, mice were killed.

\section{Immunohistochemistry}

Three-micrometres sections of formalin-fixed, paraffin-embedded primary tumours and metastatic tissues from separate patients with CRC were subjected to heat-mediated antigen retrieval in citrate buffer ( $\mathrm{pH} \mathrm{6)}$ before blocking in sequential peroxydase and protein block for $20 \mathrm{~min}$ at $20^{\circ} \mathrm{C}$. Samples were incubated with primary mouse anti-CXCR7 monoclonal antibody (IgG1 clone 11G8; R\&D Systems Europe, Lille, France) for $45 \mathrm{~min}$ at $20^{\circ} \mathrm{C}$ and then with a HRP-conjugated rabbit anti-mouse antibody.

\section{Statistical analysis}

Statistical comparisons were performed using the non-parametric Man-Whitney $U$-test, and statistical significance was set at $P<0.05$.

\section{RESULTS}

\section{CXCR7 and its ligands are over-expressed in human CRC}

To determine whether CXCR7, one of the two specific receptors for CXCL12, was expressed in colon carcinoma, we first analysed by quantitative RT-PCR the mRNA levels for CXCR7 on surgical resection pieces of human primary colorectal tumours $(n=29)$ and on healthy colon $(n=14)$ tissues. Our analysis of this independent set of samples revealed that the CXCR7 receptor was expressed in healthy human colon tissues and significantly more expressed (4.2fold) in the biopsies of CRC (Figure 1A, Supplementary Table 1). As previously described, CXCR4, the other well-characterised
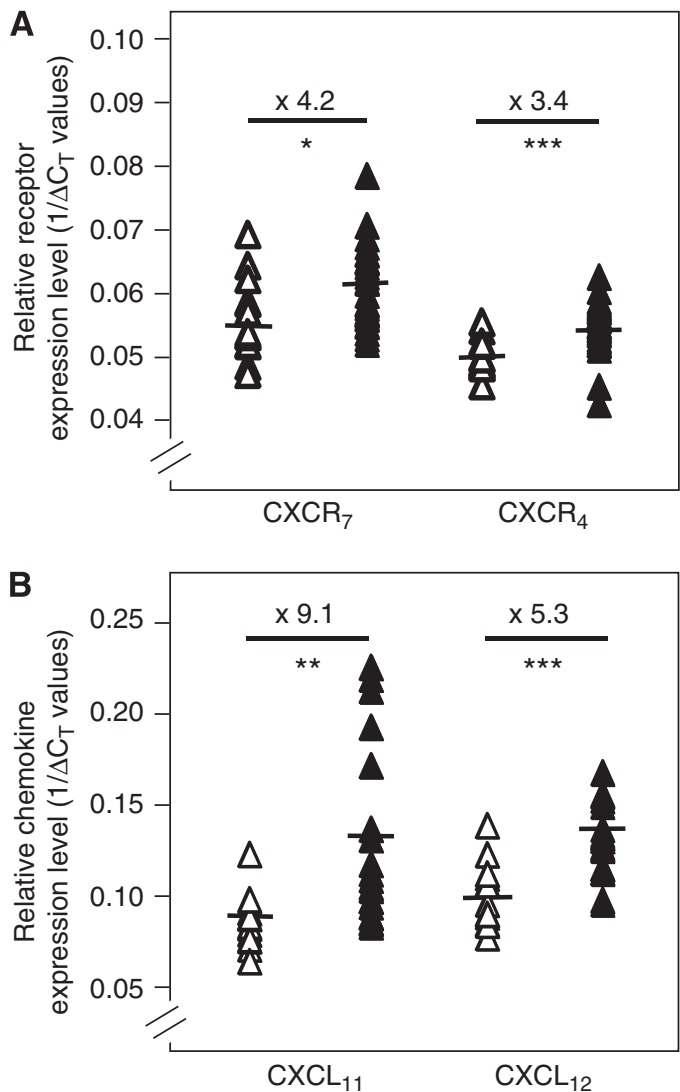

Figure I Expression of CXCR7 and its ligands in human primary colon carcinoma. Quantitative RT-PCR analysis of CXCR7 and CXCR4 receptors (A) and CXCLII and CXCLI2 chemokines (B) in surgical resection pieces of human colon carcinoma (filled symbols) compared with healthy colon tissues (open symbols). The horizontal bars indicate the median values of each group. The relative expression level of genes is calculated using human actin and GAPDH as normalising genes and expressed as $\mid /(\Delta) C_{T} .{ }^{*} P<0.01,{ }^{* *} P<0.001$ and ${ }^{* * *} P<0.000 \mid$. 
specific receptor for CXCL12, was significantly more expressed (3.4-fold) in cancer $v s$ healthy samples (Figure 1A, Supplementary Table 1).

We thus evaluated the ability of human primary colorectal tumours to produce the specific CXCR7 ligands. Analysis by quantitative RT-PCR indicated that both CXCL11 and CXCL12 chemokines were significantly more expressed (9.1- and 5.3-fold, respectively) in the biopsies of CRC compared with healthy human colon, suggesting that a potential autocrine/ paracrine relationship for the activation of CXCR7 could exist in malignant primary colorectal tumours (Figure 1B, Supplementary Table 1).

\section{In primary colorectal tumours, as well as in lung and liver metastases from patients, CXCR7 receptors are mainly} detected in the vicinity of tumour-associated blood vessels

Depending on the origin of cancer, CXCR7 was described to be expressed both by the tumour cells and the stromal cells (Hou et al, 2010; Sun et al, 2010). Little is known about the protein expression of CXCR7 in CRC. By immunohistochemistry using anti-CXCR7 specific monoclonal antibody (11G8 clone; R\&D Systems), we were then intrigued to identify CXCR7-expressing cells in primary colorectal tumours and also in lung and liver metastases.

CXCR7 protein expression was first analysed in human CRC sections from primary tumours $(n=11)$, and we observed the presence of CXCR7 in all colorectal tumours tested (Supplementary Table 2). The staining was almost exclusively detected in the cytoplasm of tumour-associated blood vessel cells (Figure 2B) but
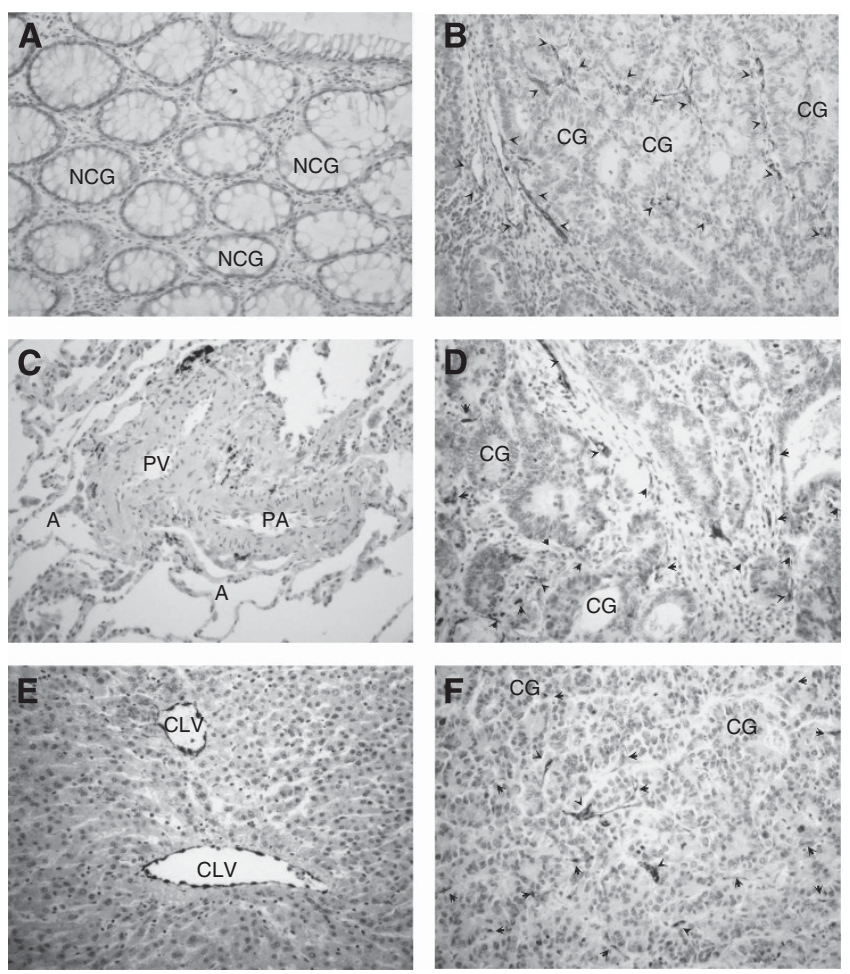

Figure 2 CXCR7 localisation in human colon carcinoma and it metastases. Detection of CXCR7 protein expression in representative specimens of primary colorectal tumour (B), metastatic lung (D) and liver (F) and corresponding non-cancerous neighbour colon $(\mathbf{A})$, lung $(\mathbf{C})$ and liver (E) tissues as assessed by immunohistochemical staining with I I G8 CXCR7-specific antibodies. Original magnification $\times 200$. Abbreviations: $\mathrm{A}=$ alveoli; $\mathrm{CG}=$ carcinomatous gland; $\mathrm{CLV}=$ centrolobular vein; $\mathrm{PA}=$ pulmonary artery; PV = pulmonary vein; NCG = non-carcinomatous gland. Arrows indicate the CXCR7 staining of tumour vessels. never in the colon cancer cells. However, most of the normal colon tissues (Figure 2A) displayed undetectable CXCR7 levels, except in some specimens where a few submucosal vessels were stained positively for CXCR7 (Supplementary Table 2).

In the lung $(n=8)$, as well as in the liver $(n=9)$, metastases from separate patients with CRC, the expression of CXCR7 was also mostly observed in the tumour-associated blood vessels and undetected in the tumour cells (Figure 2D and F). However, and more strikingly, no positive cells were found in the healthy tissue of the lungs (Figure 2C), whereas in all samples from normal liver, CXCR7 was expressed by most of the normal centrolobular veins (Figure 2E).

\section{Systemic CXCR7 antagonism reduces pre-established lung metastases but not liver metastases from colon cancer}

Based on the expression of CXCR7 in the human colon tumours and particularly in metastatic sites, we hypothesised that the blockade of CXCR7 might be an effective treatment against the CRC metastases. We tested this hypothesis by evaluating the effects of the CCX754 and CCX771 compounds, two potent specific CXCR7 antagonists (Burns et al, 2006; Zabel et al, 2009), on mouse experimental models of metastasis using mouse C26 and human HT29 colon cancer cell lines, previously characterised for their CXCR7 expression (Supplementary Figure 1).

In vivo, the treatment with CXCR7 antagonists was administered to animals (Figure $3 \mathrm{~A}$ and $\mathrm{B}$ ) at a stage where the presence of experimental micro metastases had been verified (data not shown). When the lungs were examined at killing, CXCR7 antagonisttreated mice exhibited fewer pulmonary metastases than vehicletreated mice both in the C26 and HT29 models (Figure 3C and E). Treatment of the mice with CXCR7 antagonists significantly reduced the number of metastases in the lungs of C26-injected mice (by $40 \%, 77 \pm 6$ vs $129 \pm 5$ ) and in the lungs of HT29-injected mice (by $56 \%, 2.6 \pm 0.8$ vs $5.9 \pm 1.4$ ). Surprisingly, systemic antagonism with CCX compounds did not lead to any significant decrease in tumour burden in the liver, either in the C26 or HT29 tumour models (Figure 3D and F).

\section{CXCR7 and its ligands are differentially expressed in lung} and liver metastases from colon cancer

The CXCR7/CXCR7 ligands axis has been described to be involved in paracrine interactions promoting tumour progression (Duda et al, 2011; Verbeke et al, 2011). In the light of these observations, and with the objective to understand the ineffectiveness of CXCR7 antagonist treatment on liver metastases compared with the lung's, we have been led to examine and compare the expression levels of CXCR7 and its ligands within both metastastic sites.

Using specific probes directed against human or murine CXCR7 and CXCR7 ligands, the metastatic lungs and livers from immunodeficient mice injected with human HT29 cells were exploited to quantify and to distinguish mRNAs, resulting from human malignant cells themselves from those resulting from murine stromal cells. Important differences were indeed detected in the expression of CXCR7 ligands between colon tumour cells from lung and liver metastases (Figure 4A, Supplementary Table 1). We observed significantly higher expressions of CXCL11 (12-fold increase) and CXCL12 (37-fold increase) by the human tumour cells from lung metastases compared with liver metastases. In contrast, there was no significant difference in the expression of either chemokines between the stromal cells from lung or from liver metastases (Figure 4B). Interestingly, we found that the lung tissues showed a 12-fold increase in the expression levels of murine CXCR7 mRNA compared with liver tissues (Figure 4B, Supplementary Table 1). 

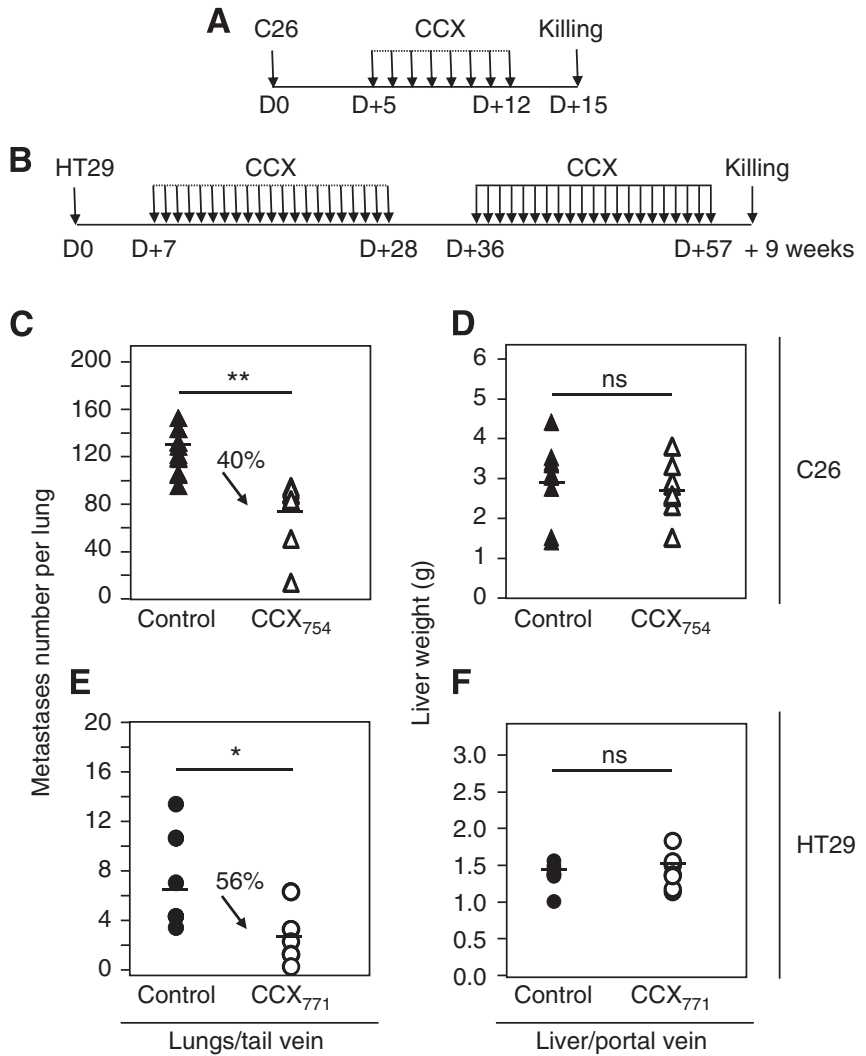

Figure 3 Effect of treatments by CXCR7 antagonists on pre-established colon cancer metastases. (A, B) Schematic representations of treatments by CXCR7 antagonists of experimental metastases from C26/BALB/C (A) and HT29/SCID (B) models. (C-F) Mice were injected with C26 cells (C, D) or with HT29 cells $(\mathbf{E}, \mathbf{F})$ into the tail vein $(\mathbf{C}, \mathbf{E})$ or into the portal vein (D, F) before receiving subcutaneous injections of CCX754, CCX77I or vehicle (control). Upon killing, the extent of tumour development was assessed. The horizontal bars indicate the median values of each group. $n=8-12$ mice per group. Abbreviation: $n s=$ not significant; $P<0.05$ and ${ }^{* *} P<0.0001$.

The VEGF and CXCL8 angiogenic factors are not under control of the CXCR7 receptors in colorectal lung metastases

The CXCR7/CXCR7 ligands axis might be directly implicated in the tumour angiogenesis but also indirectly, by regulating processes that lead up to the expression of proangiogenic factors such as CXCL8 and VEGF (Wang et al, 2008; Hou et al, 2010). Therefore, we assessed the mRNA expression of CXCL8 and VEGF in HT29injected mice treated (or not) with a CXCR7 antagonist. As shown in Figure 4C, the treatment with the CCX771 compound did not significantly affect the expression of CXCL8 or VEGF in the human malignant cells nor the expression of VEGF in the murine stromal cells (the homologous CXCL8 does not exist in mouse).

\section{DISCUSSION}

The chemokine CXCL12 has been implicated in multiple steps of tumorigenesis and progression of metastatic disease (Balkwill, 2004; Zlotnik, 2008). It was generally believed that CXCL12 mediated these processes via a single cell-surface receptor, CXCR4. Since then CXCR7 has been identified as an alternate receptor for CXCL12, and many studies have highlighted that CXCR7 receptors also have key functions in promoting the development of several types of tumours (Burns et al, 2006; Miao et al, 2007; Meijer et al, 2008; Wang et al, 2008; Bennani-Baiti et al, 2010; Grymula et al,
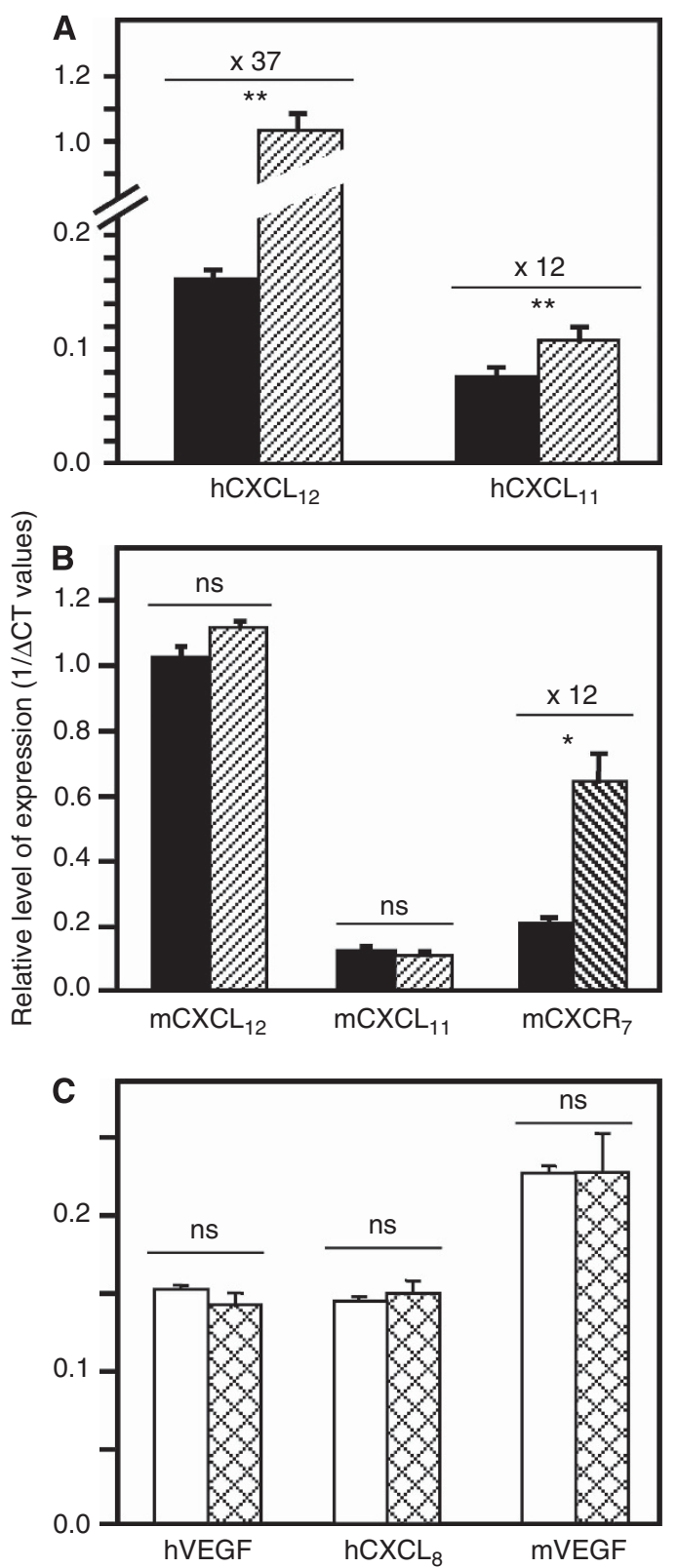

Figure 4 Expression of CXCR7 ligands, VEGF and CXCL8 within metastases of colon carcinoma. (A, B) Quantitative RT-PCR analysis of expression of CXCR7 and its ligands in metastatic liver ( $n=2$, black bars) and lung ( $n=2$, hatched bars) from HT29/SCID models using human $(\mathbf{A})$ or mouse (B) probes. (C) Quantitative RT-PCR analysis of expression of CXCL8 and VEGF in metastatic lung from not treated (empty bars) or treated by CCX77I (cross-hatched bars) HT29/Scid mice using human or mouse probes. The relative level of expression of genes is calculated using mouse raslp or human actin and GAPDH as normalising genes and expressed as $I /(\Delta) C_{T}$. Abbreviation: $n s=$ not significant; " $P<0.05$ and ${ }^{* *} P<0.005$.

2010; Hattermann et al, 2010; Kollmar et al, 2010). Consequently, tumour events regulated by CXCL12 seem more complex than previously thought, justifying the examination of earlier findings related to this chemokine in light of an additional receptor. CXCR7 expression has been reported in various human cancers, including lung, prostate, breast and liver carcinoma (Miao et al, 2007; Wang et al, 2008; Iwakiri et al, 2009; Marechal et al, 2009; Zheng et al, 2010). To date, CXCR7 has never been examined in human colon carcinoma. 
In the present study, we first evaluated the expression of CXCR7 in the human colorectal adenocarcinoma. Our results show a stronger induction of mRNA expression for this receptor in CRC compared with normal colon mucosa. At the cellular level, in the primary tumours, the presence of the CXCR7 protein was restricted to tumour-associated endothelial cells, whereas it was clearly absent in epithelial and endothelial normal cells, as well as in tumour cells. With the exception of the centrolobular veins in the healthy liver, which, surprisingly, were positive for CXCR7, the same cellular distribution of the CXCR7 protein was observed in lung and liver metastases. These results are consistent with studies in other human cancers that have also described CXCR7 expression on the neovasculature associated with tumour, but not on most of the non-transformed cells or non-tumour vasculature (Miao et al, 2007; Monnier et al, 2012). Nevertheless, one study has demonstrated, by using specific antibodies as defined by Berahovitch et al (2010), that in human biopsies of rhabdomyosarcomas, breast and lung cancers, CXCR7 was expressed on a majority of tumour-associated blood vessels but, also, on the malignant cells (Miao et al, 2007). All these observations lead to speculate that the CXCR7 expression in tumour vessels is a common feature of all cancers, whereas the presence of this receptor in malignant cells would be restricted to a few types of cancers.

Besides, our study has shown that expression of CXCL12, the common chemokine to CXCR4 and CXCR7 receptors, was significantly higher in CRC than in normal colon mucosa. This finding is consistent with previous studies where the increased expression of CXCL12 in colorectal tumours has also been significantly associated with tumour stage, lymphatic invasion, venous invasion, lymph node and distant metastases and decreased survival rate (Verbeke et al, 2011). As for the other known ligand for CXCR7, and as we previously reported (Cambien et al, 2009), our results showed a mRNA induction of CXCL11 in CRC compared with normal colon, which was confirmed at the protein level by Yang et al (2011).

Collectively, all these data suggested that the CXCR7-CXCR7 ligands axis could have key functions on the process of human colon carcinoma metastasis and prompted us to assess its in vivo relevance. With the aim of investigating if CXCR7 could also intervene in the growth of metastases of CRC, we tested whether a systemic treatment with CXCR7 antagonists, such as CCX754 or CCX771 compounds (Burns et al, 2006), could affect local growth of pre-established metastases. Interestingly, this systemic treatment by CXCR7 antagonist strongly reduced the tumour expansion within lungs of both HT29- and C26-inoculated mice. Thus, CXCR7 appeared to be a key factor in the progression of colon cancer metastases in the lungs of mice. Moreover, and in contrast to the effects on pulmonary metastases, the extent of liver metastasis was unaffected by the CXCR7 antagonist treatment, suggesting that CXCR7 may mediate the metastasis process of colon carcinoma in an organ-specific manner.

To gain further insights into an organ-specific inhibition of metastatic colon cancer growth through CXCR7 antagonism, we analysed the expression of CXCR7 and its ligands within lung and liver tumours. Investigations at mRNA level were conducted on tumours from HT29 model, which was then crucial to quantify and to distinguish mRNAs resulting from human malignant cells themselves from those resulting from murine stromal cells. Thus, our data highlighted that, in pulmonary metastatases compared with liver foci, human CRC cells expressed more CXCL11 and CXCL12, whereas murine stromal cells expressed more CXCR7 receptors. Together, these results suggested that CXCR7 ligands secreted by tumour cells could reach, in the lungs but not in the liver, levels sufficient to induce a paracrine process, which may further contribute to the progression of metastases and/or exacerbation of the disease. Moreover, knowing that CXCR7 receptors were exclusively expressed by the endothelial cells in CRC, it was tempting to speculate that the CXCR7 ligands could directly or/and indirectly regulate vascular development within lung metastases.

The CXCR7 axis is known to be indirectly implicated in tumour vascularisation through processes leading up to the expression of proangiogenic factors such as CXCL8 and VEGF (Wang et al, 2008; Hou et al, 2010). In our study, we observed that in the lung metastases of CRC, CXCR7 axis failed to regulate the expression of CXCL8 and VEGF (at the level of transcription), rather suggesting a direct control of CXCR7 axis on vascular tumour growth. In this case, as it has been reported, CXCL12 could help the angiogenesis process by stimulating the migration and division of the endothelial cells (Duda et al, 2011) and/or could facilitate the vasculogenesis process through the attraction of the endothelial progenitor cells (Dai et al, 2011; Yan et al, 2012). It should be noted that, in vivo pathological concentrations of CXCL12 alone fail to induce significant vascularisation (Mirshahi et al, 2000), whereas in contrast, in synergy with soluble factors, such as VEGF, they can be able to induce potent vascularisation (Kryczek et al, 2005). So, it is reasonable to assume that in lung metastases, unlike liver metastases, the levels of CXCL12 are sufficient to trigger these synergistic effects, thereby confirming the study by Meijer et al (2008) who suggested that CXCR7 axis may control tumour development mainly in the tissues with high CXCL12 expression. In light of these findings, we propose that the combination of antiVEGF and anti-CXCR7 strategies could be particularly effective in the treatment of lung metastasis of colon cancer.

Finally, our study suggests that chemokine expression by tumour cells is essential for metastatic development in the lungs. Nevertheless, host environment notably shapes tumour cellchemokine expression and could thereby contribute, together with intrinsic properties of colon tumour cells, to tissue specificity of metastatic process.

In summary, our data point to a distinct role of the CXCR7/ chemokines axis in lung metastasis compared with liver metastasis. Systemic treatments with CXCR7 antagonists significantly reduce metastasis of colon cancer cells in the lungs without affecting that of the liver and provide supporting evidence that targeting the CXCR7 axis may be beneficial in limiting metastatic colon cancer.

\section{ACKNOWLEDGEMENTS}

This work was supported in part by the Institut National de la Santé et de la Recherche Médicale (INSERM) and by Cancéropôle Provence Alpes Côte d'Azur (Grant: ACI 07086AA). We thank Dr Mark ET Penfolf (ChemoCentryx, Inc) for providing CXCR7 antagonists and our successful scientific discussions. We also thank all the technicians of the Animal Facility of the Centre Méditerranéen de Médecine Moléculaire, C3M (INSERM, Unit 1065, Nice, France) for the quality of their work, Nadège Gonthier of the Highspeed quantitative RT-PCR Facility of the Equipe Associée EA 6302 (CHU Archet 1, Nice France) for the excellent technical assistance and Pr Mery Tulic (University of Western Australia) for help with the English language and critical reading of the manuscript.

Supplementary Information accompanies the paper on British Journal of Cancer website (http://www.nature.com/bjc)

\section{REFERENCES}

Balabanian K, Lagane B, Infantino S, Chow KY, Harriague J, Moepps B, Arenzana-Seisdedos F, Thelen M, Bachelerie F (2005) The chemokine
SDF-1/CXCL12 binds to and signals through the orphan receptor RDC1 in T lymphocytes. J Biol Chem 280(42): 35760-35766 
Balkwill F (2004) Cancer and the chemokine network. Nat Rev Cancer 4(7): 540-550

Bennani-Baiti IM, Cooper A, Lawlor ER, Kauer M, Ban J, Aryee DN, Kovar $\mathrm{H}$ (2010) Intercohort gene expression co-analysis reveals chemokine receptors as prognostic indicators in Ewing's sarcoma. Clin Cancer Res 16(14): 3769-3778

Berahovitch R, Penfold M, Schall T (2010) Non specific CXCR7 antibodies. Immunol Lett 133: 112-114

Burns JM, Summers BC, Wang Y, Melikian A, Berahovich R, Miao Z, Penfold ME, Sunshine MJ, Littman DR, Kuo CJ, Wei K, McMaster BE, Wright K, Howard MC, Schall TJ (2006) A novel chemokine receptor for SDF-1 and I-TAC involved in cell survival, cell adhesion, and tumor development. J Exp Med 203(9): 2201-2213

Cambien B, Karimdjee BF, Richard-Fiardo P, Bziouech H, Barthel R, Millet MA, Martini V, Birnbaum D, Scoazec JY, Abello J, Al Saati T, Johnson MG, Sullivan TJ, Medina JC, Collins TL, Schmid-Alliana A, Schmid-Antomarchi H (2009) Organ-specific inhibition of metastatic colon carcinoma by CXCR3 antagonism. Br J Cancer 100(11): 1755-1764

Corbett TH, Griswold DP, Mayo JG, Laster WR, Schabel Jr FM (1975) Cyclophosphamide-adriamycin combination chemotherapy of transplantable murine tumors. Cancer Res 35(6): 1568-1573

Dai X, Tan Y, Cai S, Xiong X, Wang L, Ye Q, Yan X, Ma K, Cai L (2011) The role of CXCR7 on the adhesion, proliferation and angiogenesis of endothelial progenitor cells. J Cell Mol Med 15(6): 1299-1309

Duda DG, Kozin SV, Kirkpatrick ND, Xu L, Fukumura D, Jain RK (2011) CXCL12 (SDF1alpha)-CXCR4/CXCR7 pathway inhibition: an emerging sensitizer for anticancer therapies? Clin Cancer Res 17(8): 2074-2080

Grymula K, Tarnowski M, Wysoczynski M, Drukala J, Barr FG, Ratajczak J, Kucia M, Ratajczak MZ (2010) Overlapping and distinct role of CXCR7SDF-1/ITAC and CXCR4-SDF-1 axes in regulating metastatic behavior of human rhabdomyosarcomas. Int J Cancer 127(11): 2554-2568

Hattermann K, Held-Feindt J, Lucius R, Muerkoster SS, Penfold ME, Schall TJ, Mentlein R (2010) The chemokine receptor CXCR7 is highly expressed in human glioma cells and mediates antiapoptotic effects. Cancer Res 70(8): 3299-3308

Hou KL, Hao MG, Bo JJ, Wang JH (2010) CXCR7 in tumorigenesis and progression. Chin J Cancer 29(4): 456-459

Iwakiri S, Mino N, Takahashi T, Sonobe M, Nagai S, Okubo K, Wada H, Date H, Miyahara R (2009) Higher expression of chemokine receptor CXCR7 is linked to early and metastatic recurrence in pathological stage I nonsmall cell lung cancer. Cancer 115(11): 2580-2593

Keeley EC, Mehrad B, Strieter RM (2011) Chemokines as mediators of tumor angiogenesis and neovascularization. Exp Cell Res 317(5): 685-690

Kollmar O, Rupertus K, Scheuer C, Nickels RM, Haberl GC, Tilton B, Menger MD, Schilling MK (2010) CXCR4 and CXCR7 regulate angiogenesis and CT26.WT tumor growth independent from SDF-1. Int J Cancer 126(6): 1302-1315

Kryczek I, Lange A, Mottram P, Alvarez X, Cheng P, Hogan M, Moons L, Wei S, Zou L, Machelon V, Emilie D, Terrassa M, Lackner A, Curiel TJ, Carmeliet P, Zou W (2005) CXCL12 and vascular endothelial growth factor synergistically induce neoangiogenesis in human ovarian cancers. Cancer Res 65(2): 465-472

Kryczek I, Wei S, Keller E, Liu R, Zou W (2007) Stroma-derived factor (SDF-1/CXCL12) and human tumor pathogenesis. Am J Physiol Cell Physiol 292(3): C987-C995
Marechal R, Demetter P, Nagy N, Berton A, Decaestecker C, Polus M, Closset J, Deviere J, Salmon I, Van Laethem JL (2009) High expression of CXCR4 may predict poor survival in resected pancreatic adenocarcinoma. Br J Cancer 100(9): 1444-1451

Meijer J, Ogink J, Roos E (2008) Effect of the chemokine receptor CXCR7 on proliferation of carcinoma cells in vitro and in vivo. Br J Cancer 99(9): 1493-1501

Miao Z, Luker KE, Summers BC, Berahovich R, Bhojani MS, Rehemtulla A, Kleer CG, Essner JJ, Nasevicius A, Luker GD, Howard MC, Schall TJ (2007) CXCR7 (RDC1) promotes breast and lung tumor growth in vivo and is expressed on tumor-associated vasculature. Proc Natl Acad Sci USA 104(40): 15735-15740

Mirshahi F, Pourtau J, Li H, Muraine M, Trochon V, Legrand E, Vannier J, Soria J, Vasse M, Soria C (2000) SDF-1 activity on microvascular endothelial cells: consequences on angiogenesis in in vitro and in vivo models. Thromb Res 99(6): 587-594

Monnier J, Boissan M, L'Helgoualc'h A, Lacombe ML, Turlin B, ZucmanRossi J, Theret N, Piquet-Pellorce C, Samson M (2012) CXCR7 is upregulated in human and murine hepatocellular carcinoma and is specifically expressed by endothelial cells. Eur J Cancer 48(1): 138-148

Mukaida N, Baba T (2012) Chemokines in tumor development and progression. Exp Cell Res 318(2): 95-102

Sun X, Cheng G, Hao M, Zheng J, Zhou X, Zhang J, Taichman RS, Pienta KJ, Wang J (2010) CXCL12/CXCR4/CXCR7 chemokine axis and cancer progression. Cancer Metastasis Rev 29(4): 709-722

Verbeke H, Struyf S, Laureys G, Van Damme J (2011) The expression and role of CXC chemokines in colorectal cancer. Cytokine Growth Factor Rev 22(5-6): 345-358

Wang J, Shiozawa Y, Wang J, Wang Y, Jung Y, Pienta KJ, Mehra R, Loberg R, Taichman RS (2008) The role of CXCR7/RDC1 as a chemokine receptor for CXCL12/SDF-1 in prostate cancer. J Biol Chem 283(7): 4283-4294

Weitz J, Koch M, Debus J, Hohler T, Galle PR, Buchler MW (2005) Colorectal cancer. Lancet 365(9454): 153-165

Yan X, Cai S, Xiong X, Sun W, Dai X, Chen S, Ye Q, Song Z, Jiang Q, Xu Z (2012) Chemokine receptor CXCR7 mediates human endothelial progenitor cells survival, angiogenesis, but not proliferation. J Cell Biochem 113(4): 1437-1446

Yang S, Wang B, Guan C, Wu B, Cai C, Wang M, Zhang B, Liu T, Yang P (2011) Foxp3 + IL-17 + T cells promote development of cancer-initiating cells in colorectal cancer. J Leukoc Biol 89(1): 85-91

Zabel BA, Wang Y, Lewen S, Berahovich RD, Penfold ME, Zhang P, Powers J, Summers BC, Miao Z, Zhao B, Jalili A, Janowska-Wieczorek A, Jaen JC, Schall TJ (2009) Elucidation of CXCR7-mediated signaling events and inhibition of CXCR4-mediated tumor cell transendothelial migration by CXCR7 ligands. J Immunol 183(5): 3204-3211

Zeelenberg IS, Ruuls-Van Stalle L, Roos E (2003) The chemokine receptor CXCR4 is required for outgrowth of colon carcinoma micrometastases. Cancer Res 63(13): 3833-3839

Zheng K, Li HY, Su XL, Wang XY, Tian T, Li F, Ren GS (2010) Chemokine receptor CXCR7 regulates the invasion, angiogenesis and tumor growth of human hepatocellular carcinoma cells. J Exp Clin Cancer Res 29: 31

Zlotnik A (2008) New insights on the role of CXCR4 in cancer metastasis. J Pathol 215(3): 211-213

Zlotnik A, Burkhardt AM, Homey B (2011) Homeostatic chemokine receptors and organ-specific metastasis. Nat Rev Immunol 11(9): 597-606

This work is published under the standard license to publish agreement. After 12 months the work will become freely available and the license terms will switch to a Creative Commons Attribution-NonCommercial-Share Alike 3.0 Unported License. 